\title{
An Improved Delayed Self-Heterodyne Interferometer for Linewidth Measurements
}

\author{
Jay W. Dawson, Namkyoo Park, and Kerry J. Vahala
}

\begin{abstract}
We demonstrate a delayed self-heterodyne interferometer with a recirculating delay, in which loss is partially compensated by an erbium-doped fiber amplifier. $A$ resolution limit of $.606 \mathrm{~Hz}$ is achieved with an $11 \mathrm{~km}$ fiber delay line, as compared to $18.2 \mathrm{kHz}$ for the standard single-pass case. The possible effect of spectral broadening due to amplifier noise is considered and found to have a negligible effect on the system performance.
\end{abstract}

$\mathrm{T}$ HE delayed self-heterodyne interferometer (DSHI) has been an important tool for the measurement of laser linewidths since its conception [1]. However, the requirement that the delay time, $\tau_{D}$, exceed the coherence time of the laser, $\tau_{C}$, has limited the usefulness of the technique to lasers with relatively broad linewidths. Semiconductor lasers are the most notable of these. Recently, however, the DSHI technique has also been used to measure linewidth in fiber laser systems [2], [3]. Okamura and Iwatsuki [3] used a DHSI system with a $72 \mathrm{~km}$ fiber delay line to measure the linewidth of an erbium fiber laser. The resolution of this measurement was 1.4 $\mathrm{kHz}$ and data indicated that the measurement was still instrument-resolution limited.

Tsuchida [4] reported on an improvement to the DSHI method which uses a recirculating delay, allowing the same fiber delay to be used multiple times. By including an acoustooptic modulator as a frequency shifter in the delay arm of the recirculating DSHI (RDSHI), multiple delays could be determined by counting frequency shifts. However, due to large losses, Tsuchida was only able to measure up to three passes through the fiber delay.

We report here on a significant improvement to the RDSHI. We include in the delay arm an erbium-doped fiber amplifier. By partially compensating the large loss of the delay arm with gain from the fiber amplifier we easily discern beat notes from light that has passed through the delay as many as 30 times. An $11 \mathrm{~km}$ fiber delay line yields a resolution limit of $18.2 \mathrm{kHz}$ with a standard DSHI. Our loss-compensated RDSHI yielded a resolution limit of 606 $\mathrm{Hz}$ for the same fiber length.

The experimental setup is shown in Fig. 1. Components include an acousto-optic modulator (AOM), which pro-

Manuscript received May 18, 1992; revised June 18, 1992. This work was supported by the Northrop Corporation.

The authors are with the Department of Applied Physics, California Institute of Technology, Pasadena, CA 91125.

IEEE Log Number 9202569.

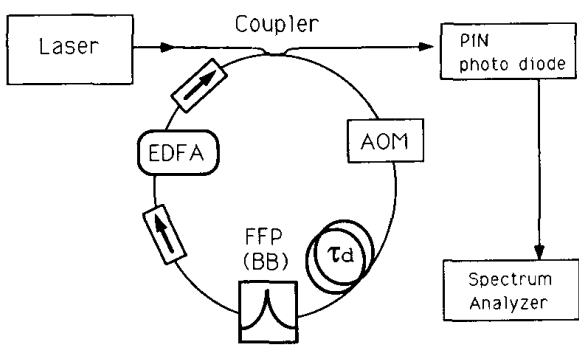

Loss compensated RDSHI

Fig. 1. Schematic of the recirculating delayed self-heterodyne interferometer with loss-compensation. EDFA: erbium-doped fiber amplifier, $\tau_{D}$ : delay line, AOM: acoustooptic modulator, FFP (BB) broadband fiber Fabry-Perot filter.

vided a frequency shift of $140 \mathrm{MHz}$ with a conversion efficiency of $10 \%$ at $1550 \mathrm{~nm}$; two fiber input/output couplers to collimate the light out of the fiber for transmission through the AOM and then to refocus it back into the fiber at the AOM output; a delay line consisting of an $11 \mathrm{~km}$ length of optical fiber having a net loss of 0.2 $\mathrm{dB} / \mathrm{km}$ in the wavelength range of interest; and a fused fiber-optic coupler with a $90 / 10$ coupling ratio. $90 \%$ of the light per pass was returned to the recirculator and $10 \%$ was sent to the photodiode. We estimate that the total loss per pass through the recirculator was $18 \mathrm{~dB}$. Light was detected with an Ortel photodiode (model 2515B) having a frequency response up to $15 \mathrm{GHz}$. This gave us the potential to see light that had been delayed by as many as 100 passes through the fiber delay line. The output of the photodiode was observed on a spectrum analyzer with a maximum bandwidth of $33 \mathrm{GHz}$.

In addition to these components which are standard in a conventional RDSHI, we added an erbium-doped fiber amplifier $(G)$ with two fiber-optic isolators having a reverse isolation of $35 \mathrm{~dB}$ each. Two commercially available amplifer units were tested. One was a germanium-only codoped gain module capable of providing $40 \mathrm{~dB}$ of small-signal gain at $1537 \mathrm{~nm}$ and a maximum saturation output power of $8.43 \mathrm{dBm}$. It could provide sufficient gain to compensate the loss in the RDSHI delay arm over a bandwidth of $6 \mathrm{~nm}$ about $1537 \mathrm{~nm}$. The other module was an aluminum-germanium codoped gain module capable of providing $37.2 \mathrm{~dB}$ of small signal gain at $1532 \mathrm{~nm}$ and a maximum saturation output power of $10.3 \mathrm{dBm}$. It could 
provide sufficient gain to compensate the loss in the RDSHI delay arm over a bandwidth of $30 \mathrm{~nm}$ from 1528 $\mathrm{nm}$ to $1558 \mathrm{~nm}$.

The light beam diffracted by the AOM closes the recirculating loop. Thus, with no RF drive power to the AOM the loop is open. With zero signal input to the RDSHI and the AOM on, strong beat notes $(>30 \mathrm{~dB}$ above the noise floor) spaced $18.2 \mathrm{kHz}$ apart (the freespectral-range of the delay arm) were seen on the spectrum analyzer. This indicated the recirculator was oscillating. This was obviously undesirable as it might interfere with the measurement.

Two steps were taken to prevent the system from oscillating. First, a fiber Fabry-Perot (FFP) filter was included in the delay arm. The FFP filter had a free-spectral-range of $32 \mathrm{~nm}$ and a $3 \mathrm{~dB}$ bandwidth of $40.2 \mathrm{GHz}$. This inhibited broadband oscillation, restricting it to the immediate spectral region surrounding the FFP filter transmission peak, which was adjusted to transmit the signal wavelength. Second, the pump power to the amplifier was adjusted downward until the signal input power was sufficient to saturate the gain to a point below the oscillation threshold. This prevented oscillation while still greatly reducing the net system loss. Taking these steps, spectrum analyzer beat notes now occurred only at the expected 140 $\mathrm{MHz}$ spacing, with none observable at the $18.2 \mathrm{kHz}$ spacing indicative of oscillation.

It was observed that loud acoustic noises were capable of broadening the measured linewidths (by a factor of about 2-5). To test whether this acoustic broadening was from the measurement system or from the test laser, the entire $11 \mathrm{~km}$ fiber length was placed in a styrofoam box to isolate it from these acoustic noises. There was no observed decrease in the laser linewidth due to this system change. However, placing the test laser in a similar box did produce a narrowing of the linewidth. Furthermore, after 22 passes through the recirculator the linewidth was observed to saturate at $4 \mathrm{kHz}$ and did not keep increasing as would be expected if the RDSHI was the cause of the broadening. We concluded that the measured broadening was due to the test laser.

In [4] the case of RDSHI in which the net system loss is greater than $6 \mathrm{~dB}$ is considered theoretically. This is clearly not the case in our system. For high recirculator loss the main contribution to the power of the $k$ th-order beat note is from the undelayed signal field beating with the signal field that has passed through the recirculator delay $k$-times. For the case of a loss-compensated recirculating delay it is necessary to consider multiple contributions to the $k$-th order beat note. (For the purposes of discussion, the case of the laser coherence time less than $k$ times the recirculator round-trip time will be considered.) In particular, the signal field that has been delayed by $n+k$ passes $(n=0,1,2 \cdots)$ can beat with a signal field that has been delayed by $n$ passes to generate a contribution to the $k$ th-order beat-note intensity. We must therefore sum over $n$, which in our case can be as large as 100 . Provided that $k \tau_{D}$ is greater than the laser

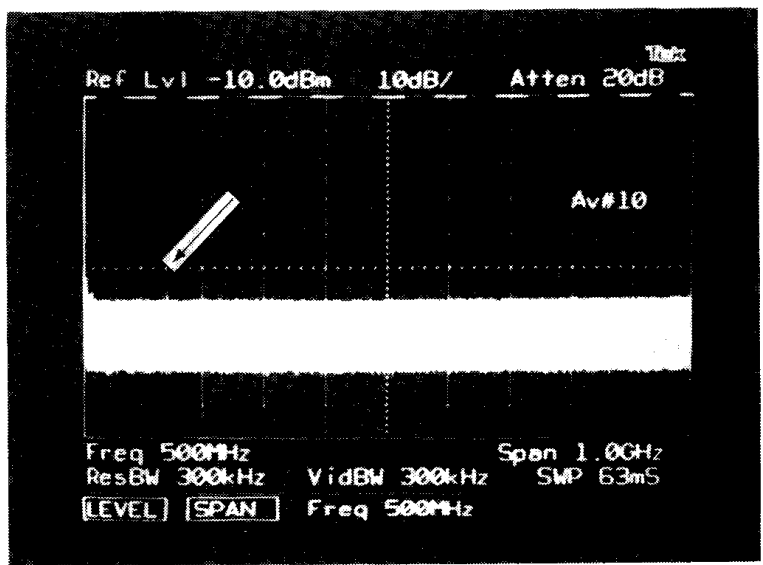

(a)

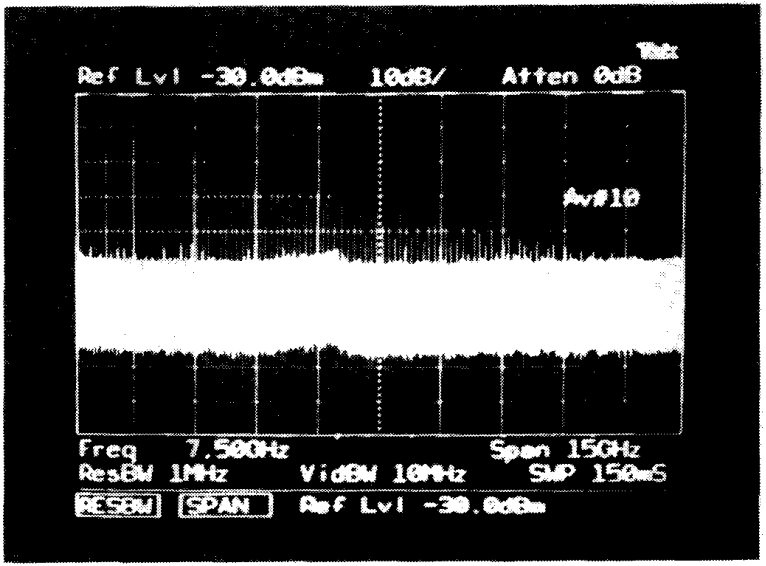

(b)

Fig. 2. Broad-band view of RDSHI photocurrent power spectrum output. (a) $1 \mathrm{GHz}$ span without amplifier, (b) $15 \mathrm{GHz}$ span with amplifier.

coherence time each of these contributions will have the same lineshape. The $k$ th beat-note under these conditions will therefore have a lineshape equivalent to a normal DSHI with the same equivalent delay time.

We used a recently developed erbium-doped fiber laser [5]-[7] capable of stable, single-frequency, widely-tunable operation combined with low intensity noise and high sidemode suppression (greater than $48 \mathrm{~dB}$ ) to test our system. Details of this laser's linewidth properties will be reported elsewhere [8]. Fig. 2 shows the photocurrent power spectra for various conditions. In Fig. 2(a) the amplifier has been removed from the recirculator. Only a few AOM beat-note peaks are seen. In Fig. 2(b) the amplifier has been inserted and many more AOM beatnote peaks are visible. We estimate that loss per pass has decreased from $18 \mathrm{~dB}$ in the uncompensated case to approximately $0.2 \mathrm{~dB}$ per pass with compensation.

Fig. 3 shows the measured FWHM linewidth of the laser as a function of order. The RDSHI resolution as a function of order is also plotted. The spectrum analyzer was used to average the linewidth over several seconds. The measured linewidth is observed to increase with in- 


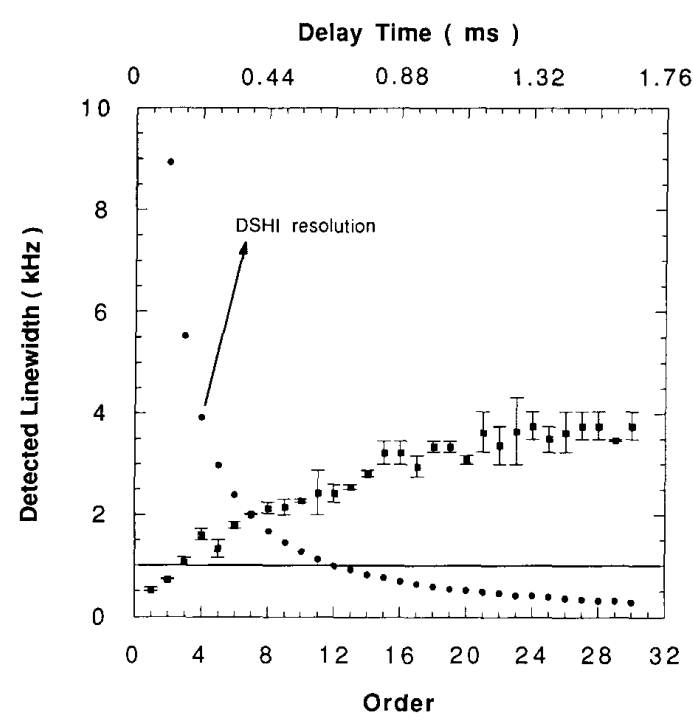

Fig. 3. Measured laser bandwidth as a function of order. RDSHI resolution as a function of order is also plotted.

creasing order and ultimately saturate at $4 \mathrm{kHz}$ around the 22nd order. This behavior is characteristic of a laser with both a short term and long term frequency stability and is discussed in [8].

However, before this peculiarity could be attributed to the fiber laser, it was necessary to eliminate the possibility of spectral broadening due to the erbium amplifier. There is apparent disagreement in the literature [3], [9], as to whether erbium-doped fiber amplifiers broaden the linewidth of a coherent source. To investigate further we removed the $11 \mathrm{~km}$ delay line from the RDSHI in order to make $k \tau_{D}$ much less than the laser coherence time [9]. The results are shown in Fig. 4. Fig. 4(a)-(c) shows the linewidth at $1 \mathrm{st}, 20 \mathrm{th}$, and 30 th orders, respectively. The maximum observed FWHM linewidth is less than $400 \mathrm{~Hz}$ indicating that spectral broadening from the amplifier should not interfere with our measurement (the resolution limit of which is $606 \mathrm{~Hz}$ at 30 orders with the $11 \mathrm{~km}$ delay line).

As a further check, we measured the FWHM linewidth for the first three orders both with and without the amplifier in place. No discernable difference in linewidth between the system with the amplifier and the system without the amplifier was observed.

In conclusion, we have demonstrated a significant improvement to the DSHI method of linewidth measurement for laser lines within the erbium-doped fiberamplifier's bandwidth. The results should be applicable to other rare-earth doped fiber amplifiers and their corresponding spectral bandwidths. We have shown that it is possible to obtain a resolution limit of $606 \mathrm{~Hz}$ with an 11 $\mathrm{km}$ fiber delay. Furthermore, we have investigated spectral broadening due to amplifier phase noise and found that it does not have a significant effect on our measurement.

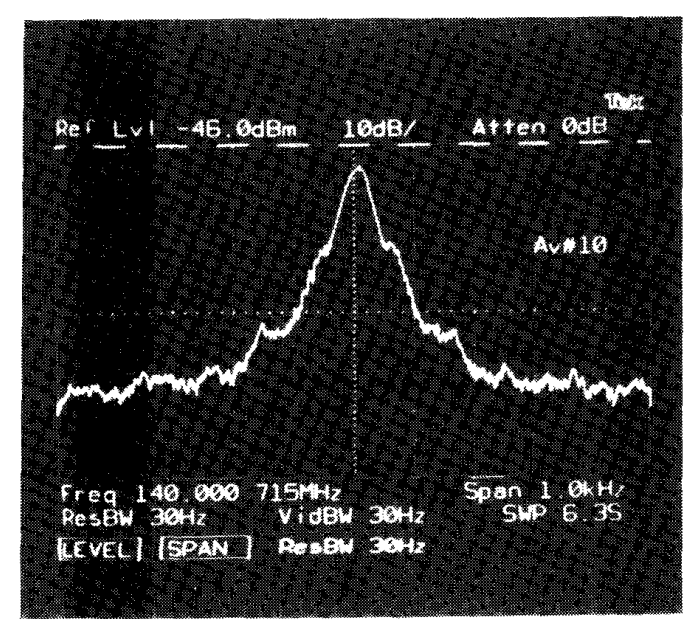

(a)

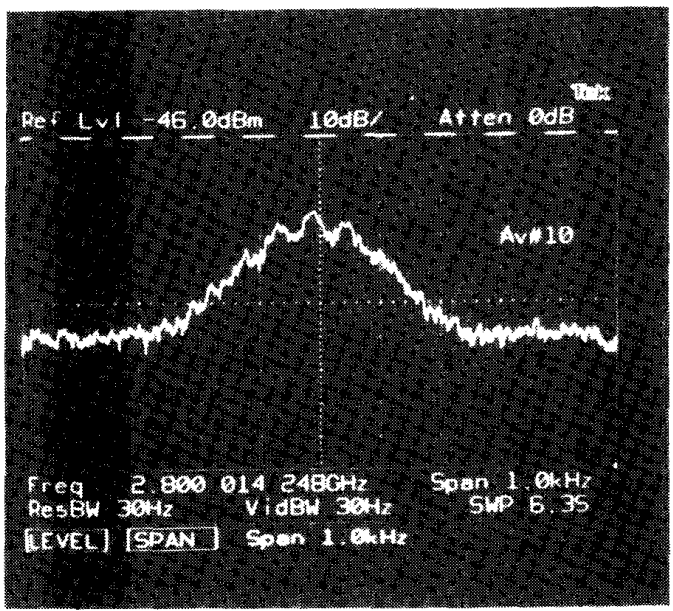

(b)

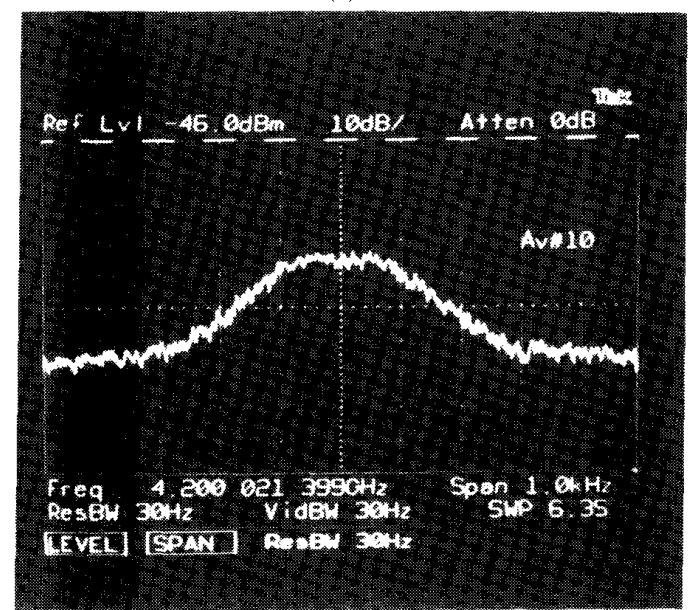

(c)

Fig. 4. RDSHI output without delay line. (a) 1st order; (b) 20th order; (c) 30 th order. 


\section{ACKNOWLEDGMENT}

We would like to thank the Ortel corporation for the loan of the photodiode used in this work.

\section{REFERENCES}

[1] T. Okoshi, K. Kikuchi, and A. Nakayama, "Novel method for high resolution measurement of laser output spectrum," Electron. Lett., vol. 16 , pp. $630-631,1980$.

[2] P. R. Morkel, G. J. Cowle, and D. N. Payne, "Travelling-wave erbium fiber ring laser with $60 \mathrm{kHz}$ linewidth," Electron. Lett., vol. 26, pp. 632-634, 1990.

[3] H. Okamura and K. Iwatsuki, "Spectral linewidth broadening in Er-doped-fiber amplifiers measured with less than $1.4 \mathrm{kHz}$ linewidth light source," Electron. Lett., vol. 26, pp. 1965-1967, 1990.

[4] H. Tsuchida, "Simple technique for improving the resolution of the delayed self-heterodyne method," Opt. Lett., vol. 15, pp. $640-642,1990$

[5] N. K. Park, J. W. Dawson, K. J. Vahala, and C. Miller, "All fiber, low threshold, widely tunable single-frequency, erbium-doped fiber ring laser with a tandem fiber Fabry-Perot filter," Appl. Phys. Lett., vol. 59, pp. 2369-2371, 1991 .

[6] J. W. Dawson, N. K. Park, K. J. Vahala, and C. Miller, "Suppression of mode-hopping in a single-longitudinal-mode erbium doped fiber laser using tandem fiber Fabry-Perot filters," Opt. Fiber Commun. Conf., OFC'92, 1992, paper WA5.

[7] S. Sanders, J. W. Dawson, N. K. Park, and K. J. Vahala, "Measurements of the intensity noise of a broadly tunable, erbium-doped fiber ring laser, relative to the standard quantum limit," Appl. Phys. Lett., vol. 60, May 25, 1992

[8] N. K. Park, J. W. Dawson, and K. J. Vahala, Opt. Lett., to be published.

[9] G. J. Cowle, P. R. Morkel, R. I. Laming, and D. N. Payne, "Spectral broadening due to amplifier phase noise," Electron. Lett., vol. 26, pp. 424-425, 1990.

\title{
Automated Measurement of Polarization Mode Dispersion Using Jones Matrix Eigenanalysis
}

\author{
B. L. Heffner
}

\begin{abstract}
Polarization mode dispersion (PMD), which can limit the bandwidth of optical transmission links, has been difficult to measure in a manner independent of human judgment, leading to difficulties in automating the measurement. We demonstrate for the first time that PMD in any linear, time invariant network can be completely characterized by eigenanalysis of Jones matrices measured at a series of discrete wavelengths, even for networks exhibiting polarization-dependent loss. A fast, automated system using a tunable laser and an accurate, real-time polarimeter affords temporal accuracy of approximately $2 \%$ down to a limit of several femtoseconds, as demonstrated by comparison with other techniques and comparison with known samples. Both the principal states of polarization and the group delay difference are measured as a function of optical frequency.
\end{abstract}

\section{INTRODUCTION}

$\mathrm{T}$ HOROUGH characterization of the optical components intended for high-speed transmission links requires accurate, repeatable measurement of polarization mode dispersion (PMD). PMD, which may limit transmission bandwidths in practical systems, is a fundamental characteristic of a network or device under test (DUT) that describes its propensity to split a narrow-band optical input pulse into two temporally separate output pulses

Manuscript received May 22, 1992.

The author is with Hewlett-Packard Laboratories, Palo Alto, CA 94303.

IEEE Log Number 9202702. according to state of polarization (SOP). PMD is completely characterized by a wavelength-dependent, threedimensional polarization dispersion vector, or equivalently by the specification of a pair of principal states of polarization (PSP) and a differential group delay $\Delta \tau$ as a function of wavelength.

Several PMD measurement techniques have been reported. Those based on changes in the auto- or cross-correlation of a low-coherence source [1] must employ a wide-spectrum source in order to achieve good temporal resolution, making them unsuitable for measurement of devices whose PMD varies with wavelength. The technique of [2], which relates $\Delta \tau$ to the density of extrema in the spectrum of transmission through the DUT in series with a polarizer, yields poor resolution in the variation of $\Delta \tau$ with wavelength and does not identify the PSP. Measurement of the arc described by the output SOP on the Poincare sphere over a series of wavelengths [3], or measurement of the frequency derivatives of normalized Stokes vectors [4], would be difficult to automate because of erroneous results produced when a measured SOP is near one of the PSP. The technique to be described suffers none of these limitations or disadvantages.

\section{THEORY}

R. C. Jones gave an explicit algorithm for experimentally determining the forward transmission Jones matrix $\boldsymbol{T}$ 